Promote the warmth of the body by the application of hot flannels, bottle or bladders of hot water, heated bricks, \&c., to the pit of the stomach, the armpits, between the thighs, and to the soles of the feet. Warm clothing may generally be obtained from bystanders.

On the restoration of life, when the power of swallowing has returned, a teaspoonful of warm water, small quantities of wine, warm brandy and water, or coffee, should be given. The patient should be kept in bed, and a disposition to sleep encouraged. During reaction large mustard plasters to the chest, and below the shoulders, will greatly relieve the distressed breathing.

II.-If from Intense Cold. Rub the body with snow, ice, or cold water. Restore warmth by slow degrees. In these accidents it is highly dangerous to apply heat too early.

III.-If from Intoxication. Lay the individual on his side on a bed, with his head raised. The patient should be induced to vomit. Stimulants should be avoided.

IV.-If from Apoplexy or from Sunstroke. Cold should be applied to the head, which should be kept well raised. Tight clothing should be removed from the neck and chest. Stimulants should be avoided.

Appearances which Generally Indicates Death. There is no breathing or heart's action ; the eyelids are generally half closed; the pupils dilated; the jaws clenched; the fingers semi-contracted; the tongue appearing between the teeth, and the mouth and nostrils are covered with a frothy mucus. Coldness, and pallor of surface increases.-British Medical Journal, Sept. $12,1863$.

\title{
In Memoriam.
}

"Ac primo quidem loco patrem meum memorandum habeo. Mihi enim idem evenit, quod Heberdeno quoque juniori accidit, qui in Harreiano quem habuit sermone piam patris sui tradidit commemorationem. Et nunc meum est, optimi, et carissimi patris columnam struere. Moveor equidem quoties memoriam revoco parentis amantissimi, patris dilectissimi, cujus cura me fovit, et disciplina instruxit. Ille autem, in schola tum temporis celeberimâ Westmonasteriensi a primis annis instructus, cùm ad gradum Medicinæ Doctoris consecutus esset, se morborum mentis curationi totum tradidit. His autem annis recentioribus quantum insanorum curandorum ratio in melius mutata fuerit quis vestrûm ignorat? Erant in Galliâ, erant in Angliâ-(Conolleius noster (liceat mihi dicere) præ cæteris) - qui insanos non tam cohibendos esse, et vinculis reprimendos, quàm summo studio, sunımâ curâ, summâ benignitate, fovendos et molliendos, et si fieri posset in sanam mentem denuo revocandos esse arbitrarentur. Hoc tantum adjicere velim, ne vobis diutius commorari videar, patrem meum hoc sibi semper proposuisse, ut, si quis morbo gravatus, consultum accederet, moribus humanis, et ratione benigmissima sibi conciliaret. Inde factum est ut xqualibus omnibus (pace vestrâ de patre meo dixerim) longè antecederet. Decessit tandem Devoniæ in illo quem sibi præ cæteris delegerat loco: propinquis amicisque carissimus; a pauperibus autem, nautis illius loci præcipuè, quam desideratus! Decessit, cùm jam per viginti annos tranquillo vite cursu usus fuerat; ut (id quod perraris contingit,) post vitam cæterorum necessitatibus sublevandis, datam, curis tandem remotis, amicis et sibi, et Deo vivere, posset, et ut ex hâc vitâ, ad æternæ vitæ munera paratior excederet."-Oratio ex Harveii Instituto in adibus Collegii Regalis Medicorum Londinensis habita die Junii xxvi, Mpccchxil, ab Alexandro J. Sulherland, M.D. Oxon., F.R.S., Coll. Reg. Med. Lond. Socio. 